\title{
Inhibition of Rac1 reverses enzalutamide resistance in castration-resistant prostate cancer
}

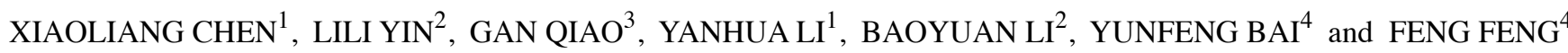 \\ ${ }^{1}$ School of Medicine; ${ }^{2}$ School of Life Sciences, Shanxi Datong University, Datong, Shanxi 037009; \\ ${ }^{3}$ School of Pharmacy, Southwest Medical University, Luzhou, Sichuan 646000; ${ }^{4}$ Department of Chemistry, \\ School of Chemistry and Environmental Engineering, Shanxi Datong University, Datong, Shanxi 037009, P.R. China
}

Received September 7, 2019; Accepted February 13, 2020

DOI: $10.3892 / \mathrm{ol} .2020 .11823$

\begin{abstract}
Enzalutamide, an androgen receptor inhibitor, has been clinically approved for the treatment of metastatic castration-resistant prostate cancer (CRPC) in the United States. However, patients only benefit from enzalutamide for a short period of time as resistance may develop. Therefore, it is vital to develop a novel strategy to overcome enzalutamide resistance. Ras-related C3 botulinum toxin substrate 1 (Rac1), which is commonly upregulated in human cancer types, has been recognized as a key molecular component in tumorigenesis, invasion and metastasis. However, the role of Rac1 in enzalutamide-resistance in prostate cancer (PCa) remains unknown. In the present study, Racl was demonstrated to be upregulated in enzalutamide-resistant $\mathrm{PCa}$ cells, and Rac1 knockdown inhibited enzalutamide-resistant cell proliferation and colony formation. Western blotting results indicated that enzalutamide treatment downregulated the expression levels of JNK and activated transcription factor 2 , as well as enhanced the $\mathrm{Bax} / \mathrm{Bcl}-2$ ratio and induced cleavage of poly-ADP ribose polymerase. Moreover, knockdown of Rac1 in MR49F cells significantly inhibited cell migration and invasion via the downregulation of Snail and the upregulation of E-cadherin. The results of a nude mouse xenograft tumor model using 22RV1 cells demonstrated that enzalutamide inhibited tumor growth after Rac1 knockdown dramatically, compared to vehicle and single treatment groups. Therefore, the present study provided novel evidence that Rac1 may serve a crucial role
\end{abstract}

Correspondence to: Professor Feng Feng or Dr Yunfeng Bai, Department of Chemistry, School of Chemistry and Environmental Engineering, Shanxi Datong University, Mingda Building, Xingyun Street, Datong, Shanxi 037009, P.R. China

E-mail: feng-feng64@263.net

E-mail: baiyunfeng1130@126.com

Key words: enzalutamide resistance, Ras-related C3 botulinum toxin substrate 1, castration-resistant prostate cancer, epithelial-mesenchymal transition in enzalutamide resistance, and that targeting Rac1 may be a potential approach for the treatment of CRPC.

\section{Introduction}

Prostate cancer ( $\mathrm{PCa})$, which is related to the activity of the androgen receptor (AR), is the second major cause of cancer-related mortality (10\%) among men in the United States in 2019 (1). Androgen deprivation therapy (ADT) is the primary approach for the treatment of $\mathrm{PCa}$, but the majority of patients eventually progress to the disease stage known as castration-resistant PCa (CRPC) (2). Enzalutamide, an AR antagonist, is currently the most effective treatment for CRPC (3). However, enzalutamide resistance develops after several months of treatment (4). Therefore, it is important to develop an effective therapeutic target to overcome enzalutamide resistance.

Rac1, a small GTPase, has been demonstrated to regulate the reorganization of the cytoskeleton during cellular activities, including cell cycle progression, migration, adhesion and polarization (5). In addition, Rac1 is commonly upregulated in ovarian cancer, breast cancer, colon cancer, and non-small cell lung cancer (6-9), and is associated with a poor prognosis. Rac1 is a key molecular component in tumorigenesis, invasion and metastasis, and directly activates various downstream pathways, including $\mathrm{p} 21$-activated kinases, actin-binding LIM kinase and cofilin, to control cellular processes $(10,11)$. Previous studies have demonstrated that Racl contributes to poor treatment response and drug resistance in cancer $(12,13)$ and Rac1 inhibition may result in a reduction in the dosage of cisplatin by 1.5 - to 3.0 -fold in head and neck squamous cell carcinoma $(14,15)$. Reinstatement of doxorubicin can sensitize squamous cell carcinoma by inhibiting Rac1 expression (16). In addition, Rac1 inhibition reverses the resistance to cisplatin and 5-fluorouracil chemotherapy in gastric adenocarcinoma spheroids and inhibits tumor growth in vivo $(17,18)$. Therefore, Rac1 may be a valid therapeutic target for patients with chemotherapy resistance.

In this study, we explored the role of Racl in the development of enzalutamide resistance and investigated the effectiveness and the associated mechanisms of combination enzalutamide and Racl depleted in CRPC. 


\section{Materials and methods}

Cell culture and lentivirus. MR49F cells were developed enzalutamide resistant cells derived from the parent cell line LNCaP, and obtained from Dr Xiaoqi Liu's lab and maintained in RPMI-1640 medium (Thermo Fisher Scientific, Inc.) containing $10 \mu \mathrm{M}$ enzalutamide at $37^{\circ} \mathrm{C}$ with $5 \% \mathrm{CO}_{2}(19)$. $\mathrm{LNCaP}$ and 22RV1 cells were purchased from the American Type Culture Collection and cultured in RPMI-1640 medium supplemented with $10 \%(\mathrm{v} / \mathrm{v})$ fetal bovine serum (FBS) (Thermo Fisher Scientific, Inc.) at $37^{\circ} \mathrm{C}$ with $5 \% \mathrm{CO}_{2}$. Lentiviruses with short hairpin RNA (shRNA) targeting Rac1 (sh-Rac1; 5'-CCTTCTTAACATCACTGTCTT-3') and non-targeting control (sh-Ctl; 5'-GCGCGATAGCGCTAA TAATTT-3') were purchased from Sigma-Aldrich; Merck KGaA. Rac1 downregulation was performed using $3 \times 10^{6}$ MR49F or 22Rv1 cells by transfecting the pLKO.1/sh-Rac1 (the titer of $10^{5}$ ) or the silencer negative control pLKO.1/sh-Ctl lentiviruses. Following transfection the cells were cultured with $2 \mu \mathrm{g} / \mathrm{ml}$ puromycin for 3 days before subsequent experiments.

Antibodies and reagents. Antibodies against Rac1 (cat. no. 2465), JNK (cat. no. 9252), activated transcription factor 2 (ATF2; cat. no. 35031), E-cadherin (cat. no. 14472), Snail (cat. no. 3879), GAPDH (cat. no. 2118), Bax (cat. no. 14796), Bcl-2 (cat. no. 4223), Ki67 (cat. no. 9129), cleaved-poly-ADP ribose polymerase (PARP; cat. no. 5625), anti-mouse IgG (cat. no. 7076), and anti-rabbit IgG (cat. no. 7074 and 4412) were purchased from Cell Signaling Technology, Inc. Enzalutamide (cat. no. S1250) was purchased from Selleck Chemicals.

Western blotting. Cells $\left(1 \times 10^{4}\right)$ were plated in $25 \mathrm{~cm}^{2}$ flask at $37^{\circ} \mathrm{C}$ with $5 \% \mathrm{CO}_{2}$. After incubation at $37^{\circ} \mathrm{C}$ for $24 \mathrm{~h}$, cells were treated with $5 \mu \mathrm{l}$ DMSO or enzalutamide $(20 \mu \mathrm{M})$ for another $48 \mathrm{~h}$. Then cells were washed thrice in ice-cold PBS, harvested and cell pellets were resuspended in RIPA lysis buffer (cat. no. R0278; Sigma-Aldrich; Merck KGaA) supplemented with a protease and phosphatase inhibitor cocktail and subjected to sonication. Sonication was performed at $4^{\circ} \mathrm{C}$ for $10-30 \mathrm{sec}$ on ice until the sample solution was clear. Protein concentrations were measured using a Pierce bicinchoninic acid protein assay kit (cat. no. 23225; Thermo Fisher Scientific, Inc.). Equal amounts of proteins (20 $\mu \mathrm{g}$ per sample) were separated using 10\% SDS-PAGE and transferred to PVDF membranes. The membranes were blocked with 5\% milk in PBS at room temperature for $1 \mathrm{~h}$ and then incubated at $4^{\circ} \mathrm{C}$ overnight with the primary antibodies, including Rac1, JNK, ATF2, E-cadherin, cleaved-PARP, Snail, GAPDH, Bax and Bcl-2 (1:1,000). Subsequently, the membranes were washed thrice with PBS and incubated with horseradish peroxidase-conjugated secondary antibodies (anti-rabbit or anti-mouse IgG; 1:2,000; Cell Signaling Technology, Inc.) for $30 \mathrm{~min}$ at room temperature. Protein bands were visualized with Super Signal West Dura Extended Duration Substrate (Thermo Fisher Scientific, Inc.). All data are representative of $\geq 3$ independent experiments.

Cell viability assay. MR49F cells $\left(3 \times 10^{3}\right.$ cells/well) were seeded into 96 -well plates, cultured for $12 \mathrm{~h}$ and treated with
$20 \mu \mathrm{M}$ enzalutamide at $37^{\circ} \mathrm{C}$ with $5 \% \mathrm{CO}_{2}$ for a certain time. MTT (10 $\mu 1,5 \mathrm{mg} / \mathrm{ml})$ was added to each well and cells were incubated for an additional $4 \mathrm{~h}$. Then, $150 \mu \mathrm{l}$ DMSO was added into each well to dissolve the formazan crystals, and the optical density at $570 \mathrm{~nm}$ was measured using a microplate reader.

Manual cell counting. MR49F cells $\left(2 \times 10^{4}\right.$ cells/well) were seeded into 6-well plates, cultured for $12 \mathrm{~h}$ and treated with $20 \mu \mathrm{M}$ enzalutamide at $37^{\circ} \mathrm{C}$ with $5 \% \mathrm{CO}_{2}$. After incubation, cells were washed thrice with PBS, harvested and counted using a TC10 automated cell counter (cat. no. 145-0010; Bio-Rad Laboratories, Inc.).

Colony formation assay. MR49F (sh-Ctl) or MR49F (sh-Rac1) cells $\left(5 \times 10^{2}\right.$ cells/well) were seeded into 6 -well plates. After incubation overnight, cells were treated with or without $10 \mu \mathrm{M}$ enzalutamide for 14 days. The colonies were fixed in $10 \%$ formalin for $15 \mathrm{~min}$ at room temperature, and stained with $1 \%$ crystal violet at room temperature for $30 \mathrm{~min}$ and the number of colonies was counted manually.

Xenograft experiments. The animal experimental protocol was approved by The Committee of Animal Experiments and Experimental Animal Welfare of Shanxi Datong University (protocol no. 2019018). Twenty male BALB/c nude mice (4-6 weeks; weighing 18-20 g; Beijing Vital River Laboratories Animal Technology Co., Ltd) were housed at a constant temperature $\left(23^{\circ} \mathrm{C}\right)$ and relative humidity $(60 \%)$ with a fixed $12 \mathrm{~h}$ light-dark cycle, and free access to food and water. After 2 weeks of feeding in the new environment, mice were divided into the four groups randomly with 5 mice per group: sh-Ctl, sh-Ctl with enzalutamide, sh-Racl, sh-Rac1with enzalutamide group. 22RV1(sh-Ctl) or 22RV1 (sh-Rac1) cells $\left(3 \times 10^{5}\right.$ cells/mouse) were mixed with $50 \%$ Matrigel gel (cat. no. 354248; Corning, Inc.) and inoculated into pre-castrated nude mice subcutaneously in the right dorsal flank until the average tumor size of sh-Ctl and sh-Ctl plus enzalutamide groups grew up to $\sim 100 \mathrm{~mm}^{3}$. The mice maintained at a specific pathogen free facility with a constant humidity and temperature at $12 / 12 \mathrm{~h}$ light/dark cycle with free access to food and water. An enzalutamide suspension $(30 \mathrm{mg} / \mathrm{kg})$ was prepared in corn oil and administered to mice in the sh-Ctl plus enzalutamide and sh-Racl plus enzalutamide groups via gavage every 2 days. The administration volume of per mouse was $0.2 \mathrm{ml}$. Of note, the coil was given every 2 days also. The tumor size and body weight were measured every 2 days by reading Vernier caliper and the tumor volumes were calculated using the formula $\mathrm{V}=\mathrm{L} \mathrm{x} \mathrm{W}^{2} / 2$, where $\mathrm{V}$ is the volume $\left(\mathrm{mm}^{3}\right)$, L is length $(\mathrm{mm})$ and $\mathrm{W}$ is the width $(\mathrm{mm})$. The wet weight and size of the tumors and body weights were measured after the the mice were sacrificed on day 24 .

Histology and immunofluorescence staining. Xenograft tumors were dissected, fixed in $10 \%$ formalin overnight at room temperature, embedded in paraffin, sectioned at $5 \mu \mathrm{m}$ thick, and mounted on poly-L-lysine-charged glass slides (cat. no. P0425-72EA; Sigma-Aldrich; Merck KGaA). The hematoxylin and eosin (H\&E) staining was completed in the Affiliated Tumor Hospital of Shanxi Datong University. 
Immunofluorescence staining was performed using an Immunodetection M.O.M kit (cat. no. PK-2200; Vector Laboratories, Inc.) according to the manufacturer's protocol. The paraffin-embedded tissue blocks were cut into $5-\mu \mathrm{M}$ sections and mounted on glass slides. After the sections were deparaffinized with xylene and rehydrated in a descending ethanol series of $70,80,90,95$ and $100 \%$, followed by antigen retrieval (cat. no. H-3300, Vector Laboratories, Inc.), microwaved on high power to $100^{\circ} \mathrm{C}$ for $3 \mathrm{~min}$. Then sections were cooled to room temperature and endogenous peroxidase activity was blocked by incubation sections with BLOXALL ${ }^{\circledR}$ Endogenous Peroxidase and Alkaline Phosphatase blocking solution (cat. no. SP-6000, Vector Laboratories, Inc.) for $10 \mathrm{~min}$ at room temperature. The sections were then incubated in working solution of M.O.M ${ }^{\mathrm{TM}}$ mouse $\mathrm{IgG}$ blocking reagent (10 $\mu \mathrm{l}$ of stock solution in M.O.M diluent) at room temperature for $1 \mathrm{~h}$. Subsequently, the sections were incubated with anti-Ki67 rabbit antibody (1:200) in M.O.M ${ }^{\mathrm{TM}}$ diluent overnight at $4^{\circ} \mathrm{C}$. After washing by PBS with $0.2 \%$ Triton $\mathrm{X}-100$, the sections were incubated with the anti-rabbit IgG (Alexa Fluor $^{\circledR} 488$ conjugate, 1:200) at room temperature for $1 \mathrm{~h}$. After washing, the sections were subsequently stained with DAPI (cat. no. P36931, Thermo Fisher Scientific, Inc.) at room temperature for $15 \mathrm{~min}$, and covered with coverslip. the sample sections were viewed using a light microscope (Axioplan 2; Zeiss, Berlin, Germany).

Cancer cell invasion and migration assay. MR49F cells $\left(4 \times 10^{4}\right)$ were seeded into the upper chamber of a 24 -well Transwell plate (cat. no. 3422; Corning, Inc.). After incubation at $37^{\circ} \mathrm{C}$ for $4 \mathrm{~h}$, the cells in the upper chamber were treated with or without $10 \mu \mathrm{M}$ enzalutamide in RPMI-1640 medium containing $0.1 \%$ FBS, and RPMI-1640 medium with $0.5 \%$ FBS, as a chemoattractant, was added into the lower chamber. Following incubation at $37^{\circ} \mathrm{C}$ for $72 \mathrm{~h}$, cells that migrated into the opposite side of the membrane were washed with PBS, fixed in $4 \%$ paraformaldehyde at room temperature for $30 \mathrm{~min}$ and stained with $0.25 \%$ crystal violet for $15 \mathrm{~min}$ at room temperature. Cell migration was quantified by counting the number of migrated cells in microscopic fields (magnification, $\mathrm{x} 100)$ per filter by the light microscope, and the mean value per filter was calculated from three replicate filters.

For the Transwell invasion assay, the upper well of the Transwell insert was precoated with $50 \mu \mathrm{l}(1 \mu \mathrm{g} / \mathrm{ml})$ Matrigel (cat. no. 356234; BD Biosciences) at $37^{\circ} \mathrm{C}$ in a $5 \% \mathrm{CO}_{2}$ incubator for $4 \mathrm{~h}$, and the MR49F cells $\left(4 \times 10^{4}\right)$ were seeded into the upper chamber. The subsequent methodology of the assay was performed as aforementioned.

Statistical analysis. Statistical analyses were performed using GraphPad Prism 7 software (GraphPad Software, Inc.), and the data are presented as the mean \pm SD. One-way ANOVA followed by Tukey's multiple comparison test was used to determine statistical significance. $\mathrm{P}<0.05$ was considered to indicate a statistically significant difference.

\section{Results}

Identification of highly expressed Racl in enzalutamideresistant PCa cell lines (19). To investigate the molecular

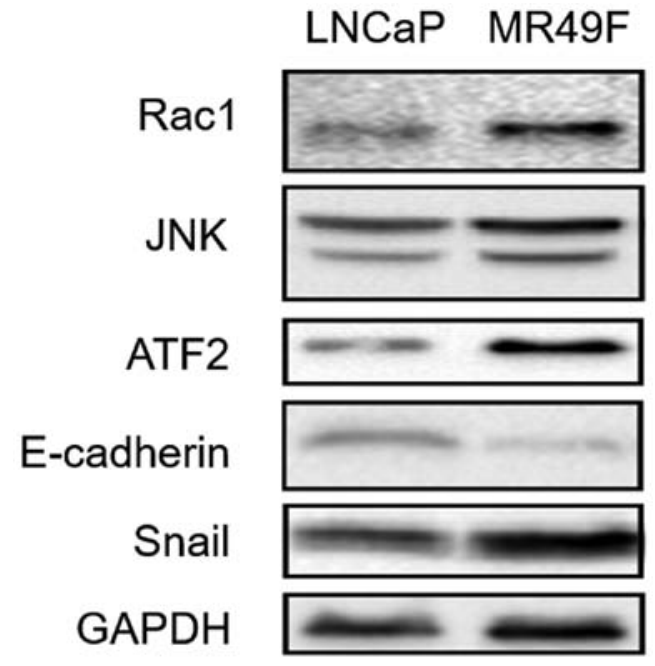

Figure 1. Rac1 upregulation in enzalutamide-resistant PCa cell lines. Rac1 expression in PCa cells after treatment with $10 \mu \mathrm{M}$ enzalutamide for 2 days was analyzed by western blotting. Rac1; Ras-related C3 botulinum toxin substrate 1 ; PCa, prostate cancer.

mechanisms underlying enzalutamide resistance in CRPC, western blotting was performed using LNCaP and MR49F cells. The results demonstrated that Rac1, JNK and ATF2 protein expression levels were upregulated in MR49F cells compared with those in LNCaP cells (Fig. 1). In addition, the protein expression levels of E-cadherin, an epithelial-mesenchymal transition (EMT) marker (20), were lower in the enzalutamide resistance cells. In contrast, Snail, another EMT marker, was much higher in MR49F cells compared with LNCaP cells. These results suggested that the Rac1/JNK/ATF2 signaling pathway may be activated in enzalutamide-resistant PCa cells.

Racl knockdown enhances the sensitivity of enzalutamideresistant PCa cells to enzalutamide. To further assess the role of Racl in the development of enzalutamide resistance in CRPC, MR49F cells were infected with a lentivirus expressing shRNA to knock down Rac1 (Fig. 2A). The results demonstrated that the treatment of Rac1-knockdown MR49F cells with enzalutamide significantly inhibited cell proliferation, compared with monotherapy with enzalutamide or Rac1-deleted (Fig. 2B and C). To clarify whether there was Rac1-independent enzalutamide resistance, the rates of LNCaP cell proliferation following enzalutamide treatment with were compared with that of Racl-knockdown MR49F cells treatment with enzalutamide. The results suggested that there were no significant difference between the two cell types (Fig. 2D). Additionally, treatment with enzalutamide also significantly inhibited colony formation in Rac1-knockdown MR49F cells compared to Rac1-deleted MR49F cells and MR49F (sh-Ctl) treated with enzalutamide alone (Fig. 2E and F). Of note, treatment of Rac1-knockdown MR49F cells with enzalutamide significantly induced cleaved-PARP and Bax expression, and downregulated Bcl-2 expression (Fig. 3E). Collectively, the present results suggested that knockdown of Racl was sufficient to induce the re-sensitization of enzalutamide-resistant PCa cells to enzalutamide.

Knockdown of Racl attenuates the migration and invasion of enzalutamide-resistant PCa cells. The effects of knocking 
A

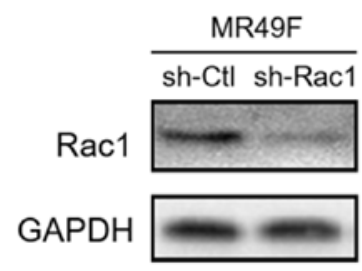

B

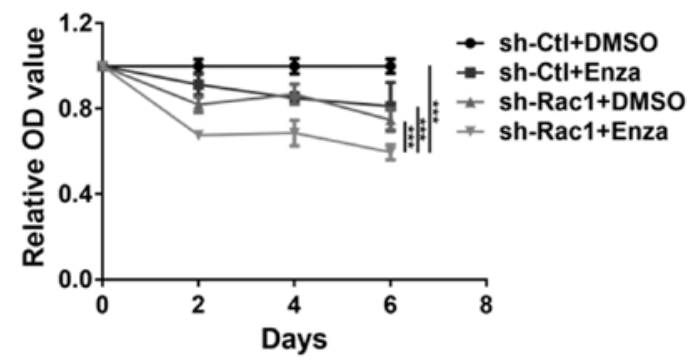

D

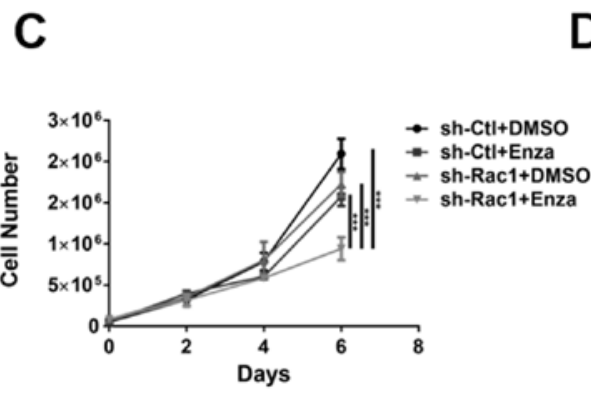

E

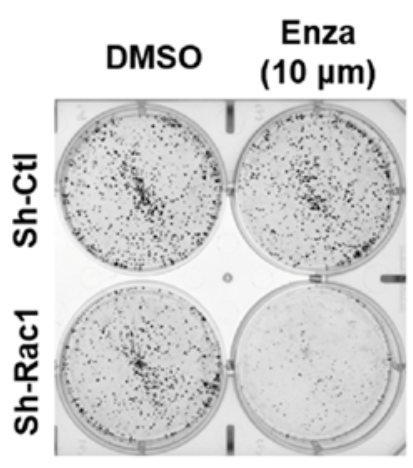

$\mathbf{F}$
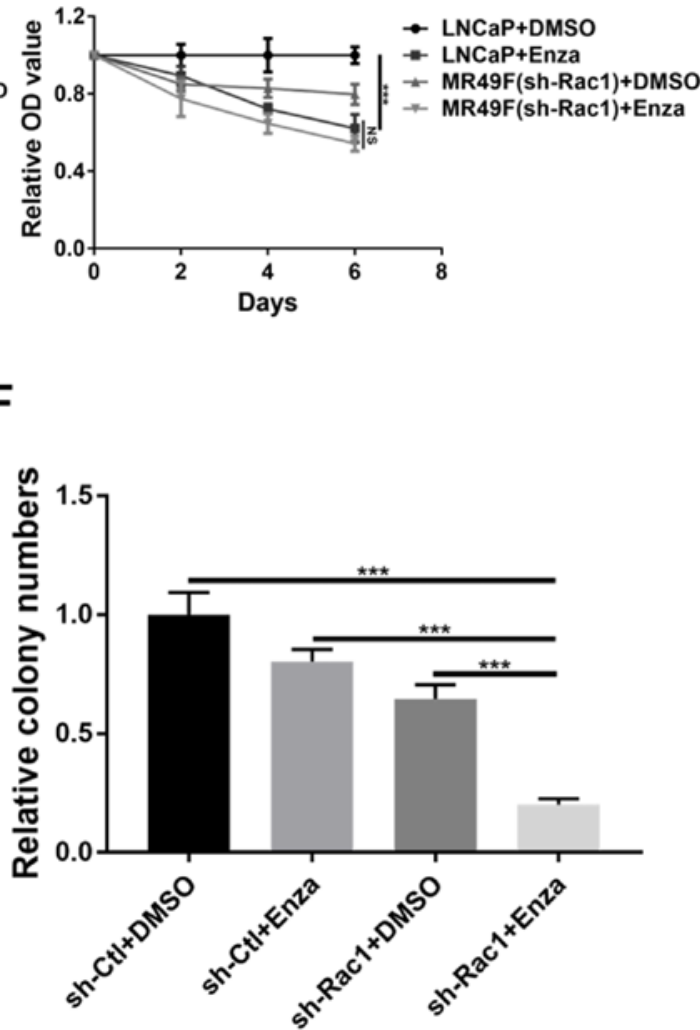

Figure 2. Rac1 knockdown enhances the sensitivity of Enza-resistant prostate cancer cells to Enza. (A) Expression of Rac1 in MR49F cells transfected with the Rac1 knockdown lentivirus. (B) MR49F cells were treated with $20 \mu \mathrm{M}$ Enza and harvested for MTT assays. (C) MR49F cells were treated with 20 $\mu \mathrm{M}$ Enza and harvested to count cell numbers. (D) LNCaP or MR49F cells were treated with $20 \mu \mathrm{M}$ Enza and harvested for MTT assays. (E) MR49F cells were treated with $10 \mu \mathrm{M}$ Enza for 2 weeks, which was followed by crystal violet staining for the colony formation assay. (F) Quantification of the colonies presented as the mean \pm SD of three independent experiments. ${ }^{* * *} \mathrm{P}<0.001$. Rac1, Ras-related C3 botulinum toxin substrate 1; shRNA, short hairpin RNA; Ctl, control; Enza, enzalutamide.

down Rac1 on MR49F cell migration and invasion were examined. The results demonstrated that enzalutamide treatment significantly inhibited the migratory and invasive abilities of MR49F cells following Rac1 knockdown (Fig. 3A-D). To study the underlying mechanisms of the enzalutamide-induced inhibitory effect on cell migration and invasion in enzalutamide-resistant PCa cells, western blotting was performed; treatment with enzalutamide in Rac1-knockdown MR49F cells upregulated of E-cadherin expression to a higher degree compared with Rac1-knockdown cells treated with DMSO or sh-Ctl-transfected cells treated with enzalutamide (Fig. 3E). Furthermore, Snail expression was decreased in
Rac1-knockdown cells treated with enzalutamide compared with the other groups. In addition, the expression levels of the downstream proteins of Rac1, including JNK and ATF2, were also decreased in Rac1-knockdown MR49F cells treated with enzalutamide. Therefore, these results suggested that knocking down Racl attenuated the migratory and invasive abilities of enzalutamide-resistant PCa cells via the upregulation of E-cadherin and downregulation of Snail expression.

Depletion of Racl enhances the efficacy of enzalutamide in enzalutamide-resistant xenograft tumors. To assess the effects of enzalutamide on the regulation of Rac1 in vivo, a 
A

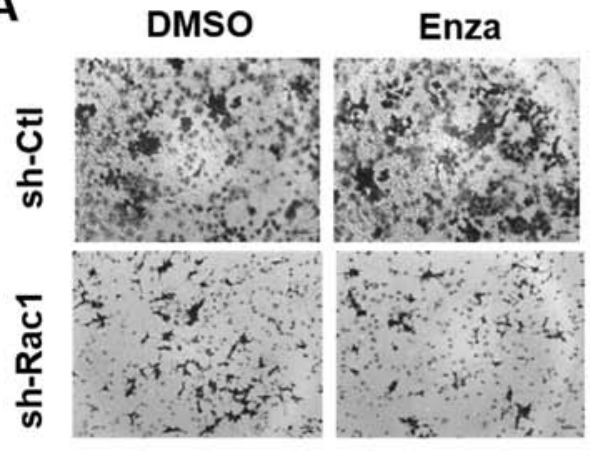

C
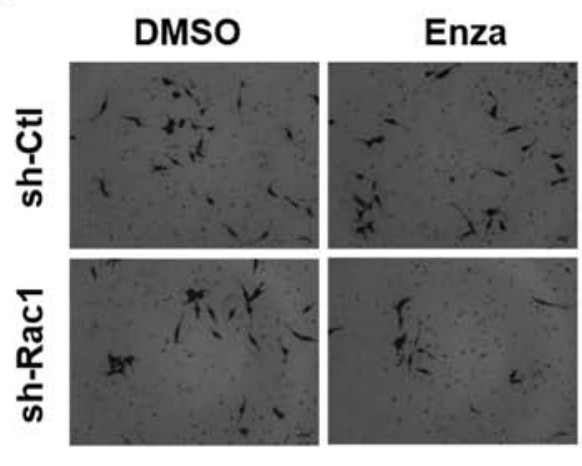

B

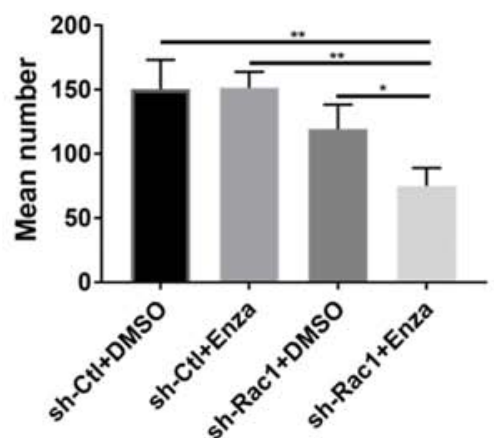

D

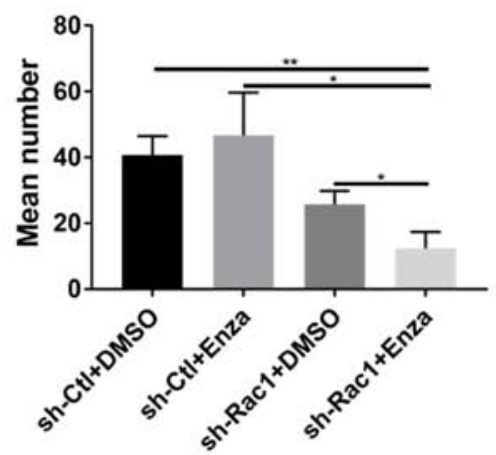

E

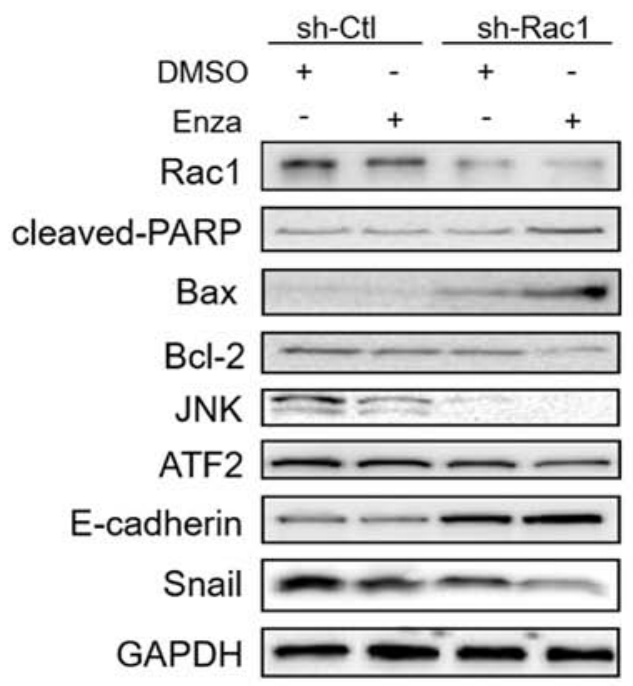

Figure 3. Knockdown of Rac1 attenuates migration and invasion of Enza-resistant PCa cells. (A-D) MR49F cells were depleted of Rac1 using an shRNA lentivirus and then treated with or without $10 \mu \mathrm{M}$ Enza followed by analysis of cell (A and B) migration and (C and D) invasion using Transwell assay at x100 magnification. (B and D) Quantification of the number of (B) migration and the invasion (D) cells. (E) Western blotting was used to assess the mechanism of the enzalutamide-induced inhibitory effect on apoptosis and EMT. Data are presented as the mean $\pm \mathrm{SD}$ of three independent experiments. $\mathrm{P}<0.05$, ${ }^{* *} \mathrm{P}<0.01$. Rac1, Ras-related C3 botulinum toxin substrate 1; shRNA, short hairpin RNA; Ctl, control; Enza, enzalutamide; ATF2, activated transcription factor 2.

22RV1-derived xenograft mice model was established(Fig.4A). The results demonstrated that Rac1 depletion inhibited tumor growth in Rac1-knockdown 22RV1 cell xenograft mice, indicating that knockdown of Racl reduced enzalutamide resistance and restored cell sensitivity to enzalutamide (Fig. 4B and C). While both wet weights and sizes of the tumors were reduced by Rac1 knockdown alone (sh-Rac1 group) or monotherapy of enzalutamide (sh-Ctl plus enzalutamide group), the effect was more significant with Rac1 knockdown plus enzalutamide treatment (Fig. 4D and E). On day 24, the mean tumor volume was reduced in Rac1-knockdown 22RV1 cell xenograft mice treated with enzalutamide $\left(365.047 \pm 157.164 \mathrm{~mm}^{3}\right)$ compared with the control mice $\left(1,221.633 \pm 509.891 \mathrm{~mm}^{3}\right.$; Fig. 4E). In addition, the volume of largest tumor was in the monotherapy of enzalutamide group $\left(3,501.142 \mathrm{~mm}^{3}\right)$. However, no significant differences were observed in the body weights of mice among the different groups (Fig. 4F), suggesting that the side effects were not aggravated when using a combined treatment of Rac1 knockdown and enzalutamide.

$H \& E$ staining of the xenograft tumors identified numerous mitotic cells in tumor tissues in the control group, suggesting that cell proliferation was active (Fig. 5A). By contrast, a 
A

B

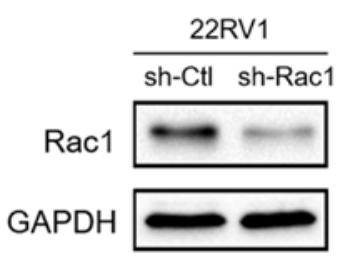

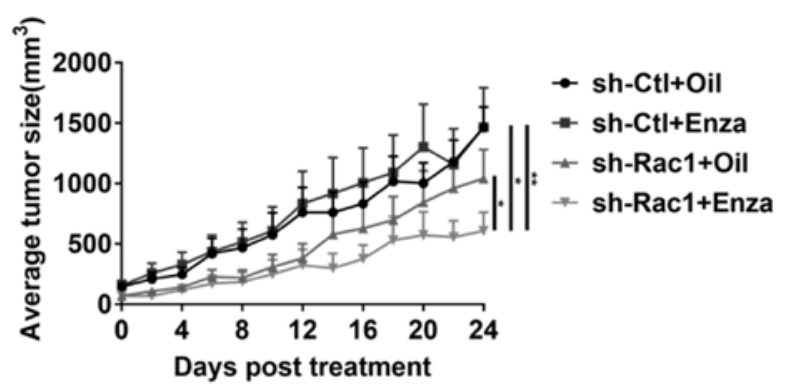

C

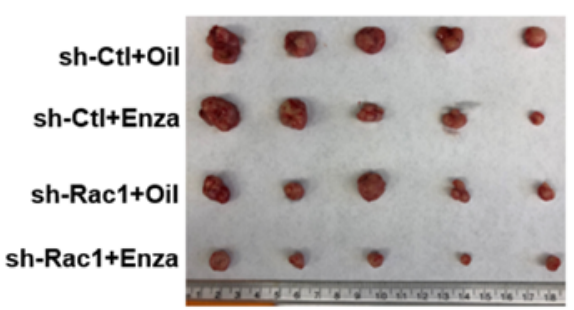

$E$

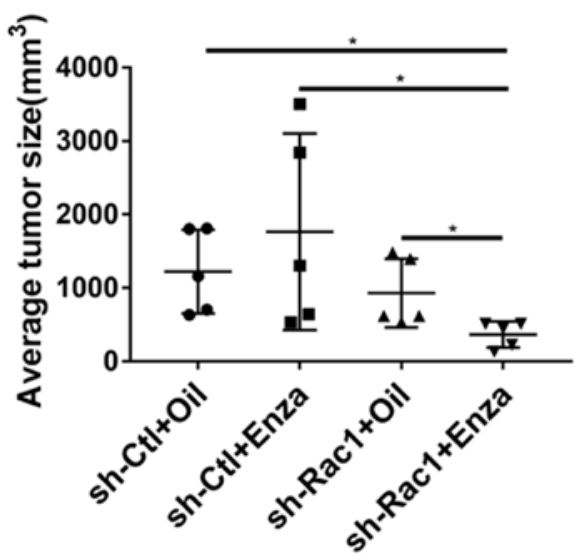

$\mathbf{F}$
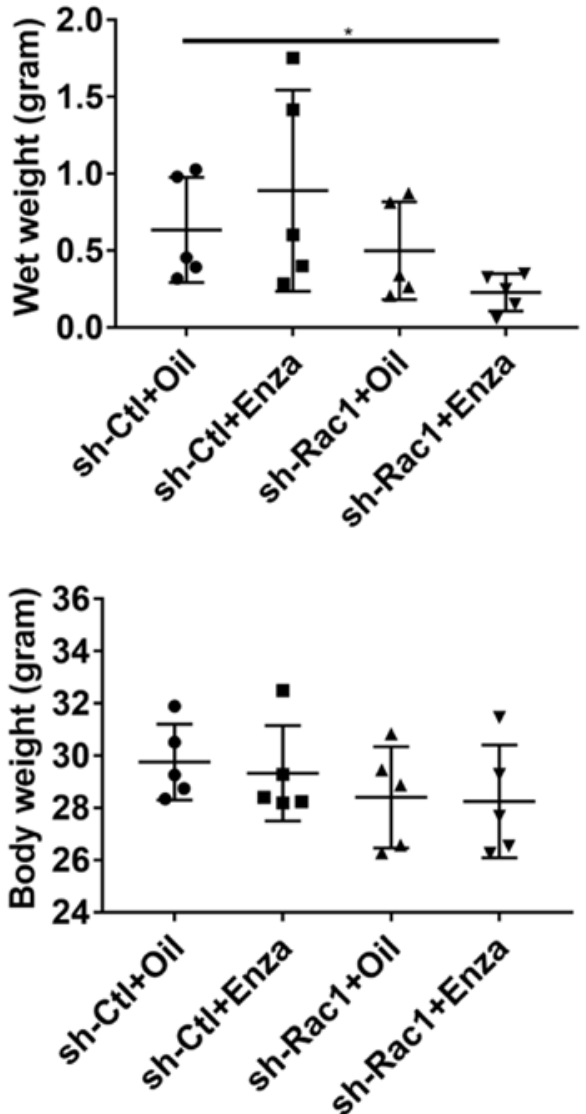

Figure 4. Depletion of Rac1 enhances the efficacy of Enza in enzalutamide-resistant xenograft tumors. (A) Castrated nude mice bearing 22RV1 (sh-Ctl)- or 22RV1 (sh-Rac1)-derived tumors were treated with Enza ( $30 \mathrm{mg} / \mathrm{kg}$ ) by oral gavage for 24 days. (B) Tumor volumes were measured every 2 days. Data are presented as the mean $\pm \mathrm{SD}, \mathrm{n}=5$ mice/group. (C) Images of the 22RV1-derived xenograft tumors at the end of study. (D) Weight and (E) volumes of the tumors were measured after mice were sacrificed. (F) Body weights were measured on day 24 prior to sacrifice. ${ }^{*} \mathrm{P}<0.05,{ }^{* *} \mathrm{P}<0.01$. Rac1, Ras-related C3 botulinum toxin substrate 1; shRNA, short hairpin RNA; Ctl, control; Enza, enzalutamide.

large number of apoptotic bodies with condensed cytoplasm and pyknotic nuclei were detected in Racl knockdown cell-derived tumors from mice treated with enzalutamide. To determine whether Rac1 knockdown alone or its combination with enzalutamide suppressed tumor proliferation and promoted apoptosis, tumor sections were analyzed by immunofluorescence staining with Ki67 (Fig. 5B and C). Immunofluorescence staining showed that the positive ratios of the Ki-67 protein in the Rac1-delepted plus the enzalutamide group were $50.893 \pm 10.221 \%$, which is less than the control $(87.001 \pm 6.915 \%)$, the enzalutamide $(85.092 \pm 5.997 \%)$ and the Rac1-depleted groups (72.465 $\pm 8.313 \%)$. Collectively, the in vivo results, which were in line with the cell-based experiments, suggested that Rac1 knockdown reversed enzalutamide-resistance in CRPC, and thus provided a novel therapeutic strategy for the treatment for CRPC.

\section{Discussion}

$\mathrm{PCa}$ is one of the most common cancer types among men in the United States (1), and enzalutamide, which was approved by the US Food and Drug Administration in 2018, is the most 
A

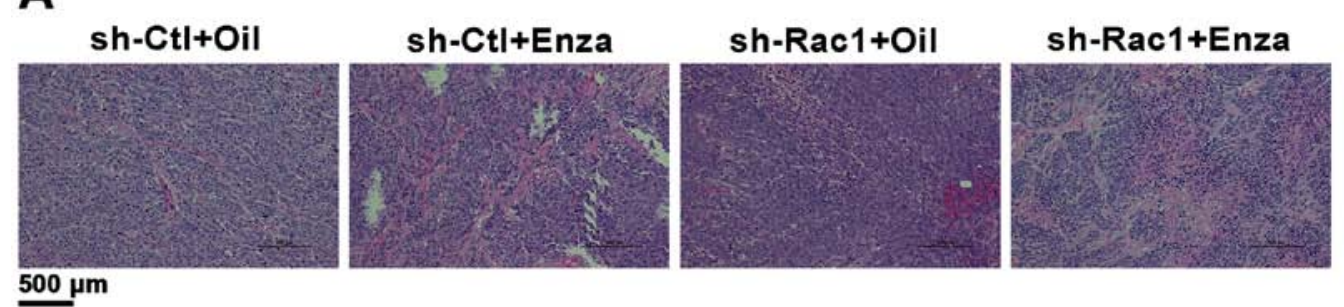

\section{B}
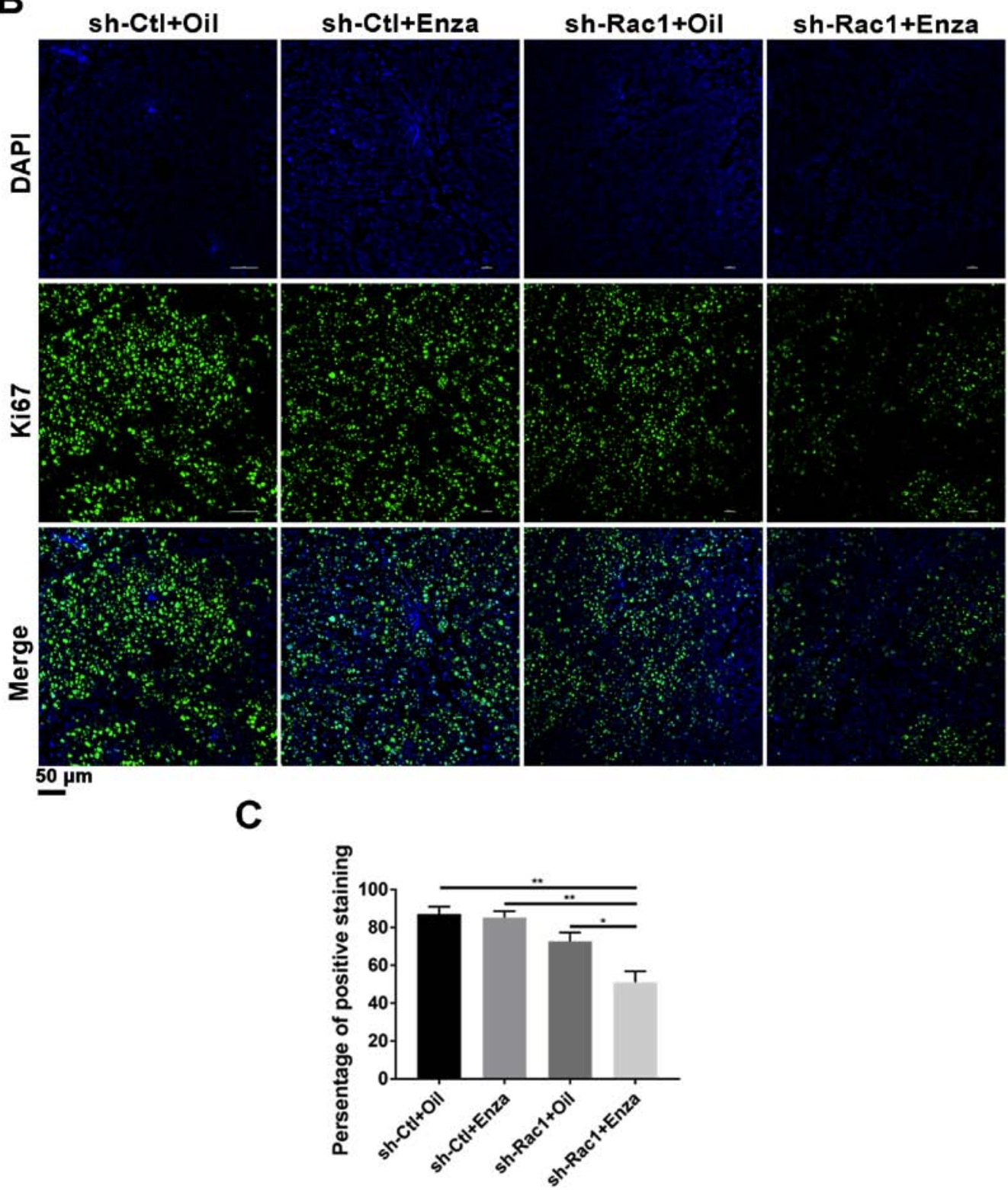

Figure 5. Pathological images of tumor sections. (A) Representative hematoxylin and eosin staining of tumor tissues. Scale bar, $500 \mu \mathrm{m}$. (B) Representative images of tumor sections showing Ki67 immunofluorescence staining. Scale bar, $50 \mu \mathrm{m}$. (C) Microscopic quantification of Ki67-positive cells expressed as the percentage of Ki67-positive cells out of the total number of cells counted. ${ }^{*} \mathrm{P}<0.05,{ }^{* * *} \mathrm{P}<0.01$. Ki67, marker of proliferation Ki-67; Rac1, Ras-related C3 botulinum toxin substrate 1; shRNA, short hairpin RNA; Ctl, control; Enza, enzalutamide.

frequently used first-line treatment for patients with metastatic CRPC (21). However, the development of enzalutamide resistance limits the utilization of enzalutamide for treating patients with CRPC (22-24). Multiple signaling pathways have been reported to contribute to enzalutamide resistance, and inhibiting these pathways has been demonstrated to enhance enzalutamide efficacy (19,25-28). Rac1 serves a major role in the regulation of cytoskeleton organization and cell adhesion, and its upregulation is associated with cancer and poor prognosis $(11,29,30)$. Furthermore, Rac1 can induce EMT by enhancing the expression of JNK $(11,29,30)$. The results of the present study suggested that Rac1 was upregulated in enzalutamide-resistant MR49F cells, thus indicating that abnormal Racl may be a key contributing 
factor in enzalutamide resistance. Furthermore, knockdown of Rac1 re-sensitized MR49F cells to enzalutamide, which prevented further cell proliferation and colony formation. Knockdown of Rac1 enhanced the efficacy of enzalutamide in enzalutamide-resistant xenograft tumors. Mechanistically, it was demonstrated that knocking down Racl inhibited the expression levels of downstream proteins JNK and ATF2. Therefore, the present study provided a potential strategy for the treatment of patients with enzalutamide-resistant CRPC. Future studies will examine the effects of Racl knockdown on the phosphorylation of JNK and assess whether activation of the Rac1/JNK signaling pathway may serve a role in the acquisition of enzalutamide resistance in CRPC cells.

EMT is a complex biological process during which epithelial cells acquire the metastatic and invasive phenotypes of mesenchymal cells $(31,32)$. EMT is highly associated with drug resistance, which results in the failure of standard chemotherapy (33). Previous studies have also revealed that EMT induces enzalutamide resistance in $\mathrm{PCa}$ (34-36). In addition, it has been demonstrated that EMT is associated with the upregulation of Rac1 $(8,17)$. Leng et al (9), have reported that Rac1 upregulation promotes EMT via the PAK1 and p38/MAPK pathways, resulting in poor prognosis. The activation of the Rac $1 / \beta$-catenin signaling pathway can also lead to cell invasion by regulating the expression levels of Snail and matrix metalloproteinase-9 (37) and inhibiting the Rac1/ $\beta$-catenin pathway that suppresses cell EMT $(38,39)$. The results of the present study indicated that the knockdown of Racl resulted in the downregulation of Snail and the upregulation of E-cadherin expression, suggesting that Rac1 promoted cell invasion and migration that in turn resulted in drug resistance. Therefore, targeted depletion of Rac1 may enhance the efficacy of enzalutamide in enzalutamide-resistant cells.

In conclusion, the results of the present study suggested that the aberrant expression of Rac1 contributed to enzalutamide resistance. In addition, the proliferation of enzalutamide-resistant cells was significantly inhibited by Racl knockdown combined with enzalutamide in vivo and in vitro. Therefore, this novel combinatorial therapeutic strategy may be used to overcome enzalutamide resistance in CRPC.

\section{Acknowledgements}

The authors would like to thank Dr Xiaoqi Liu of the University of Kentucky for providing technical support.

\section{Funding}

The study was supported by the Ph.D. Initiation Grant of Shanxi Datong University (grant nos. 2017-B-21 and 2018B-16), the Crosswise Tasks of the First Affiliated Hospital of Shanxi Datong University (grant no. HX-201938), the Natural Science Foundation of Shanxi (grant nos. 201801D121035, 201901D211427 and 201901D211428) and the Scientific and Technological Innovation Programs of Higher Education Institutions in Shanxi (grant no. 2019L0735).

\section{Availability of data and materials}

All data generated or analyzed during the present study are included in this published article.

\section{Authors' contributions}

XC, LY and FF designed the experiments. XC, LY, YB, GQ, YL and $\mathrm{BL}$ conducted the experiments. $\mathrm{XC}$ wrote the manuscript. YL, BL and FF provided technical support and supervised the progress of the experiments. XC and LY analyzed the statistical data and prepared the figures. All authors have read and approved the final manuscript.

\section{Ethics approval and consent to participate}

The present study was approved by The Committee of Animal Experiments and Experimental Animal Welfare of Shanxi Datong University (protocol no. 2019018), Datong, Shanxi, P. R. China.

\section{Patient consent for publication}

Not applicable.

\section{Competing interests}

The authors declare that they have no competing interests.

\section{References}

1. Siegel RL, Miller KD and Jemal A: Cancer statistics, 2019. CA Cancer J Clin 69: 7-34, 2019.

2. Hotte SJ and Saad F: Current management of castrate-resistant prostate cancer. Curr Oncol 17 (Suppl 2): S72-S79, 2010.

3. Siemens DR, Klotz L, Heidenreich A, Chowdhury S, Villers A, Baron B, van Os S, Hasabou N, Wang F, Lin P and Shore ND: Efficacy and safety of enzalutamide vs bicalutamide in younger and older patients with metastatic castration resistant prostate cancer in the TERRAIN trial. J Urol 199: 147-154, 2018.

4. Beer TM, Armstrong AJ, Rathkopf DE, Loriot Y, Sternberg CN, Higano CS, Iversen P, Bhattacharya S, Carles J, Chowdhury S, et al: Enzalutamide in metastatic prostate cancer before chemotherapy. N Engl J Med 371: 424-433, 2014.

5. Cardama GA, Alonso DF, Gonzalez N, Maggio J, Gomez DE, Rolfo C and Menna PL: Relevance of small GTPase Rac1 pathway in drug and radio-resistance mechanisms: Opportunities in cancer therapeutics. Crit Rev Oncol Hematol 124: 29-36, 2018.

6. Lin $\mathrm{Y}, \mathrm{Fu} \mathrm{F}, \mathrm{Lv} \mathrm{J}$, Wang $\mathrm{M}$, Li $\mathrm{Y}$, Zhang $\mathrm{J}$ and Wang $\mathrm{C}$ : Identification of potential key genes for HER-2 positive breast cancer based on bioinformatics analysis. Medicine (Baltimore) 99: e18445, 2020.

7. Xia L, Lin J, Su J, Oyang L, Wang H, Tan S, Tang Y, Chen X, Liu W, Luo X, et al: Diallyl disulfide inhibits colon cancer metastasis by suppressing Rac1-mediated epithelial-mesenchymal transition. Onco Targets Ther 12: 5713-5728, 2019.

8. Zhou Y, Liao Q, Han Y, Chen J, Liu Z, Ling H, Zhang J, Yang W, Oyang L, Xia L, et al: Racl overexpression is correlated with epithelial mesenchymal transition and predicts poor prognosis in non-small cell lung cancer. J Cancer 7: 2100-2109, 2016.

9. Leng R, Liao G, Wang H, Kuang J and Tang L: Rac1 expression in epithelial ovarian cancer: Effect on cell EMT and clinical outcome. Med Oncol 32: 329, 2015.

10. Bid HK, Roberts RD, Manchanda PK and Houghton PJ: RAC1: An emerging therapeutic option for targeting cancer angiogenesis and metastasis. Mol Cancer Ther 12: 1925-1934, 2013.

11. Engers R, Ziegler S, Mueller M, Walter A, Willers R and Gabbert HE: Prognostic relevance of increased Rac GTPase expression in prostate carcinomas. Endocr Relat Cancer 14: 245-256, 2007.

12. Hof bauer SW, Krenn PW, Ganghammer S, Asslaber D, Pichler U, Oberascher K, Henschler R, Wallner M, Kerschbaum H, Greil R and Hartmann TN: Tiam1/Rac1 signals contribute to the proliferation and chemoresistance, but not motility, of chronic lymphocytic leukemia cells. Blood 123: 2181-2188, 2014. 
13. Liu W, Chen G, Sun L, Zhang Y, Han J, Dai Y, He J, Shi S and Chen B: TUFT1 Promotes triple negative breast cancer metastasis, stemness, and chemoresistance by up-regulating the Rac1/ $\beta$-catenin pathway. Front Oncol 9: 617, 2019.

14. Arnold CR, Abdelmoez A, Thurner G, Debbage P, Lukas P, Skvortsov S and Skvortsova II: Rac1 as a multifunctional therapeutic target to prevent and combat cancer metastasis. Oncoscience 1: 513-521, 2014.

15. Skvortsov S, Dudás J, Eichberger P, Witsch-Baumgartner M, Loeffler-Ragg J, Pritz C, Schartinger VH, Maier H, Hall J, Debbage $\mathrm{P}$, et al: Rac1 as a potential therapeutic target for chemo-radioresistant head and neck squamous cell carcinomas (HNSCC). Br J Cancer 110: 2677-2687, 2014.

16. Hazar-Rethinam M, Merida de Long L, Gannon OM, Boros S, Vargas AC, Dzienis M, Mukhopadhyay P, Saenz-Ponce N, Dantzic DDE, Simpson F and Saunders NA: RacGAP1 is a novel downstream effector of E2F7-dependent resistance to doxorubicin and is prognostic for overall survival in squamous cell carcinoma. Mol Cancer Ther 14: 1939-1950, 2015.

17. Yoon C, Cho SJ, Chang KK, Park DJ, Ryeom SW and Yoon SS: Role of Racl pathway in epithelial-to-mesenchymal transition and cancer stem-like cell phenotypes in gastric adenocarcinoma Mol Cancer Res 15: 1106-1116, 2017.

18. Zeng RJ, Zheng CW, Gu JE, Zhang HX, Xie L, Xu LY and Li EM: RAC1 inhibition reverses cisplatin resistance in esophageal squamous cell carcinoma and induces downregulation of glycolytic enzymes. Mol Oncol 13: 2010-2030, 2019.

19. Farah E, Li C, Cheng L, Kong Y, Lanman NA, Pascuzzi P, Lorenz GR, Zhang Y, Ahmad N, Li L, et al: NOTCH signaling is activated in and contributes to resistance in enzalutamide-resistant prostate cancer cells. J Biol Chem 294: 8543-8554, 2019.

20. Aruga N, Kijima H, Masuda R, Onozawa H, Yoshizawa T, Tanaka M, Inokuchi S and Iwazaki M: Epithelial-mesenchymal transition (EMT) is correlated with patient's prognosis of lung squamous cell carcinoma. Tokai J Exp Clin Med 43: 5-13, 2018.

21. Wen L, Valderrama A, Costantino ME and Simmons S: Real-world treatment patterns in patients with castrate-resistant prostate cancer and bone metastases. Am Health Drug Benefits 12: 142-149, 2019.

22. Antonarakis ES, Lu C, Wang H, Luber B, Nakazawa M, Roeser JC, Chen Y, Mohammad TA, Chen Y, Fedor HL, et al: AR-V7 and resistance to enzalutamide and abiraterone in prostate cancer. N Engl J Med 371: 1028-1038, 2014.

23. Althaus A and Kibel A: Words of wisdom. Re: Enzalutamide in metastatic prostate cancer before chemotherapy. Eur Urol 67: $174,2015$.

24. Hu R, Lu C, Mostaghel EA, Yegnasubramanian S, Gurel M, Tannahill C, Edwards J, Isaacs WB, Nelson PS, Bluemn E, et al: Distinct transcriptional programs mediated by the ligand-dependent full-length androgen receptor and its splice variants in castration-resistant prostate cancer. Cancer Res 72: 3457-3462, 2012.

25. Kong Y, Cheng L, Mao F, Zhang Z, Zhang Y, Farah E, Bosler J, Bai Y, Ahmad N, Kuang S, et al: Inhibition of cholesterol biosynthesis overcomes enzalutamide resistance in castration-resistant prostate cancer (CRPC). J Biol Chem 293: 14328-14341, 2018.

26. Zhang Z, Cheng L, Li J, Farah E, Atallah NM, Pascuzzi PE, Gupta S and Liu X: Inhibition of the wnt/ $\beta$-catenin pathway overcomes resistance to enzalutamide in castration-resistant prostate cancer. Cancer Res 78: 3147-3162, 2018.
27. Bai Y, Zhang Z, Cheng L, Wang R, Chen X, Kong Y, Feng F, Ahmad N, Li L and Liu X: Inhibition of enhancer of zeste homolog $2(\mathrm{EZH} 2)$ overcomes enzalutamide-resistance in castration-resistance prostate cancer. J Biol Chem 294: 9911-9923, 2019.

28. Chen X, Liu J, Cheng L, Li C, Zhang Z, Bai Y, Wang R, Han T, Huang C, Kong Y, et al: Inhibition of noncanonical Wnt pathway overcomes enzalutamide resistance in castration-resistant prostate cancer. Prostate 80: 256-266, 2020.

29. Shin S, Buel GR, Nagiec MJ, Han MJ, Roux PP, Blenis J and Yoon SO: ERK2 regulates epithelial-to-mesenchymal plasticity through DOCK10-dependent Rac1/FoxO1 activation. Proc Natl Acad Sci USA 116: 2967-2976, 2019.

30. Zhao J, Jie Q, Li G, Li Y, Liu B, Li H, Luo J, Qin X, Li Z and Wei Y: Racl promotes the survival of $\mathrm{H} 9 \mathrm{c} 2$ cells during serum deficiency targeting JNK/c-JUN/Cyclin-D1 and AKT2/MCL1 pathways. Int J Med Sci 15: 1062-1071, 2018.

31. Brabletz T, Kalluri R, Nieto MA and Weinberg RA: EMT in cancer. Nat Rev Cancer 18: 128-134, 2018.

32. Mohammadinejad R, Biagioni A, Arunkumar G, Shapiro R, Chang KC, Sedeeq M, Taiyab A, Hashemabadi M, Pardakhty A, Mandegary A, et al: EMT signaling: Potential contribution of CRISPR/Cas gene editing. Cell Mol Life Sci 2020 (ahead of print).

33. Raoof S, Mulford IJ, Frisco-Cabanos H, Nangia V, Timonina D, Labrot E, Hafeez N, Bilton SJ, Drier Y, Ji F, et al: Targeting FGFR overcomes EMT-mediated resistance in EGFR mutant non-small cell lung cancer. Oncogene 38: 6399-6413, 2019.

34. Tucci M, Zichi C, Buttigliero C, Vignani F, Scagliotti GV and Di Maio M: Enzalutamide-resistant castration-resistant prostate cancer: Challenges and solutions. OncoTargets Ther 11: 7353-7368, 2018.

35. Liu Q, Tong D, Liu G, Xu J, Do K, Geary K, Zhang D, Zhang J, Zhang Y,Li Y, et al: Metformin reverses prostate cancer resistance to enzalutamide by targeting TGF- $\beta 1 /$ STAT3 axis-regulated EMT. Cell Death Dis 8: e3007, 2017.

36. Ware KE, Somarelli JA, Schaeffer D, Li J, Zhang T, Park S, Patierno SR, Freedman J, Foo WC, Garcia-Blanco MA and Armstrong AJ: Snail promotes resistance to enzalutamide through regulation of androgen receptor activity in prostate cancer. Oncotarget 7: 50507-50521, 2016.

37. Fan M, Xu Y, Hong F, Gao X, Xin G, Hong H, Dong L and Zhao X: Rac1//-catenin signalling pathway contributes to trophoblast cell invasion by targeting snail and MMP9. Cell Physiol Biochem 38: 1319-1332, 2016.

38. Li X, Zhu J, Liu Y, Duan $\mathrm{C}$, Chang $\mathrm{R}$ and Zhang $\mathrm{C}$ : MicroRNA-331-3p inhibits epithelial-mesenchymal transition by targeting ErbB2 and VAV2 through the Rac1/PAK1/ $\beta$-catenin axis in non-small-cell lung cancer. Cancer Sci 110: 1883-1896, 2019.

39. Du GS, Qiu Y, Wang WS, Peng K, Zhang ZC, Li XS, Xiao WD and Yang H: Knockdown on aPKC-ı inhibits epithelial-mesenchymal transition, migration and invasion of colorectal cancer cells through Rac1-JNK pathway. Exp Mol Pathol 107: 57-67, 2019.

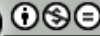

This work is licensed under a Creative Commons Attribution-NonCommercial-NoDerivatives 4.0 International (CC BY-NC-ND 4.0) License. 